\title{
Percepción cultural de los pobladores sobre el Programa Social Juntos en el Valle del Mantaro
}

Cultural perception of the inhabitants about the Juntos Social Program in the Mantaro Valley

Aparicio Chanca Flores'

Judith Ávila Mendoza²

Morelia Yolanda Aliaga Caynicela ${ }^{3}$

RECIBIDO: OCTUBRE 282019

ACEPTADO: NOVIEMBRE 152019

\footnotetext{
1 Antropólogo, Doctor en Ciencias ambientales y desarrollo sostenible, Docente, Universidad Nacional del Centro del Perú, achanca@uncp.edu.pe. ORCID. https://orcid.org/0000-00025664-5444

2 Estudiante, Facultad de Antropología, Universidad Nacional del Centro del Perú, judithavilamendoza@gmail.com, https://orcid.org/0000-0002-2598-2595

3 Estudiante, Facultad de Antropología, Universidad Nacional del Centro del Perú, bellesthorne@hotmail.com.pe, https://orcid.org/0000-0001-8051-9782
} 


\section{Resumen}

Juntos es un programa de transferencia condicionada que forma parte de la estrategia nacional Crecer; está orientado a mejorar la calidad de vida de los peruanos más necesitados e impulsar el desarrollo del capital humano, brindando mejores servicios de salud, educación y nutrición. Este estudio tuvo como objetivo conocerlapercepción cultural de los pobladores no beneficiarios sobre el Programa Social Juntos en el Valle del Mantaro. La investigación fue de tipo básico y nivel descriptivo. Las unidades de análisis consideradas fueron los pobladores no beneficiarios, tanto de género masculino como femenino, de las cuatro provincias que conforman el Valle del Mantaro: Concepción, Chupaca, Huancayo y Jauja. Mediante un muestreo probabilístico y de corte transversal, se seleccionó una muestra de 382 personas mayores de 15 años, a quienes se les administró un cuestionario tipo escala de Likert, con una fiabilidad de 0.86 según el alfa de Cronbach. Para el procesamiento y análisis de datos, se utilizó la estadística descriptiva, obteniendo como resultado que las percepciones culturales de los pobladores no beneficiarios sobre el Programa Juntos son positivas; la mayoría de los encuestados perciben aspectos positivos en la ejecución de Juntos, pues este viene repercutiendo en el cambio de actitudes y costumbres de los beneficiarios referidas a las actividades de nutrición, salud y educación.

Palabras clave: percepción, percepción cultural, programas sociales, calidad de vida.

\section{Abstract}

Juntos is a conditional transfer program, part of the national strategy Crecer; it is aimed at improving the quality of life of the most needy Peruvians and promoting the development of human capital, providing better services of health, education and nutrition. This study had as objective to know the cultural perception the non-beneficiary inhabitants from Mantaro Valley have about the Juntos program. The research was of basic type and descriptive level. The analysis units considered were the non-beneficiary inhabitants, both male and female, from the four provinces of the Mantaro Valley: Concepción, Chupaca, Huancayo and Jauja. Using a probabilistic and cross-sectional sampling, it was determined a sample of 382 people over 15 years old, who 
were administered a Likert scale questionnaire. For the processing and analysis of data, descriptive statistics were used, obtaining as a result that the cultural perceptions non-beneficiary inhabitants have about the Juntos program are positive; most respondents perceive positive aspects in the execution of Juntos since this has been having an impact on the change of the beneficiaries' attitudes and customs regarding nutrition, health and education activities.

Keywords: perception, cultural perception, social programs, quality of life.

\section{Introducción}

Un programa de transferencia condicionada, como señalan Cecchini y Madariaga (2015), consiste en la entrega de recursos monetarios y no monetarios a familias que se encuentran en situación de pobreza o pobreza extrema. Estas familias están condicionadas al cumplimiento de algunos compromisos y acuerdos con el Estado, relacionados a la mejora de sus capacidades humanas (Rico, Cecchine y Vargas, 2014; Vargas, 2014).

Estos programas, refiere Rodríguez (2011), se han impuesto en América Latina como la principal forma de intervención de los gobiernos para atender a las poblaciones más necesitadas, brindándoles ingresos monetarios.También, están orientados a mejorar las condiciones de vida de los beneficiarios (Rico et al., 2014), a la erradicación de la pobreza en la región, con igualdad y respeto a los principios de los derechos humanos (García, 1990). La mayoría de ellos son desarrollados por el Estado, que tiene la responsabilidad de atender las necesidades insatisfechas de sus habitantes (nutrición, salud y educación). De este modo, un gobierno puede poner en marcha planes, programas o proyectos que busquen garantizar el acceso a la educación, campañas de prevención para cuidar la salud o iniciativas para combatir la desnutrición infantil, entre otros (Guabloche y Sánchez, 2011). Por ello, en todo el mundo se observan muchos y variados programas sociales creados según sus necesidades y contextos situacionales (Lucci, 2018).

En el caso peruano, el Programa Juntos es uno de los programas de transferencia condicionada que se vienen ejecutando a nivel nacional; su finalidad es aliviar la pobreza actual. Guabloche y Sánchez (2011) lo consideran una inversión 
orientada a fortalecer el capital humano. El Programa Juntos es promovido y ejecutado por el Ministerio de Desarrollo e Inclusión Social (MIDIS), cuya misión fundamental es promover el acceso a los servicios de nutrición, salud y educación de las familias más pobres del país, con el objetivo de generar capital humano (MIDIS, 2017). El Programa Nacional de Apoyo Directo a los Más Pobres, denominado Juntos, fue creado en el año 2005 mediante el Decreto Supremo N. ${ }^{\circ}$ 032-2005-PCM, modificado posteriormente por el Decreto Supremo N. ${ }^{\circ}$ 062-2005-PCM (MIDIS, 2013).

Juntos es una unidad ejecutora cuyo fin es que tanto gestantes, niñas, niños, adolescentes y jóvenes hasta los 19 años de edad accedan informados a servicios de salud, nutrición y educación. Su objetivo, que se corresponde con el del MIDIS, es "contribuir con el alivio de la pobreza y potenciar el capital humano de los hogares en situación de pobreza y pobreza extrema" y contrarrestar la transmisión intergeneracional de la pobreza, alineándose a la Estrategia Nacional de Desarrollo e Inclusión Social (ENDIS): "incluir para crecer", como programa social de los ejes 1, 2 y 3 de la misma, pues contribuye a la generación de mayores oportunidades económico-sostenibles de los hogares rurales en proceso de inclusión.

La investigación permitió conocer la percepción cultural que tienen los pobladores no beneficiarios sobre el Programa Juntos en el Valle del Mantaro. Los resultados contribuirán, como señala Morera (1995), a los tomadores de decisiones de las entidades públicas y privadas que operan en esta zona. También permitirán mejorar las políticas sociales y el replanteo de los programas sociales (Segovia y Mira, 2014).

Por otra parte, cabe señalar que, en las últimas décadas, el estudio sobre percepción viene siendo de mucho interés en el campo de la antropología (Surrallés, 2003). Sin embargo, este interés ha dado lugar a problemas conceptuales; así, según Vargas (1994), "el término percepción ha llegado a ser empleado indiscriminadamente para designar a otros aspectos que también tienen que ver con el ámbito de la visión del mundo de los grupos sociales", como es la cosmovisión tanto a nivel individual y/o colectiva (Chanca, 2016). La percepción corresponde al plano de las actitudes, los valores sociales o las creencias que han sido creadas y recreadas por los seres humanos como una forma de apropiación subjetiva de la realidad. Por lo 
señalado, la percepción, para fines del presente estudio, está vinculada al sentir, pensar y actuar de los pobladores asentados en el valle del Mantaro, en relación al Programa Juntos.

En nuestro país, la pobreza constituye un grave problema que afecta a un amplio sector de la población y que tiene consecuencias económicas, políticas y sociales; impacta en la vida de las personas, limitando sus capacidades naturales, sus oportunidades de desarrollo y el goce de derechos básicos inherentes a su condición humana (Estrada y Perea, 2008; Rodríguez, 2011). Por todo ello, los programas sociales, como sostienen Vivaldi y Barra (2012), son elementos protectores de los más necesitados.

Según Cecchini y Atuesta (2017), "varias evaluaciones de impacto han demostrado que los PTC han logrado mejorar el bienestar de la población pobre en diversos aspectos tales como el ingreso, el consumo de alimentos y el acceso a educación y salud, entre otros" (p. 9). En el contexto peruano, Alcázar (2009) refiere que el Programa Juntos ha logrado resultados positivos y significativos en sus áreas de intervención. Ha generado mejoras en la salud y educación de los niños(as) así como en las prácticas de cuidado de salud y alimentación infantil (Cavero-Arguedas, De la Vega y Cuadra-Carrasco, 2017). Con el Programa Juntos, se busca atenuar la pobreza extrema, sentando bases para generar capacidades humanas y así prevenir la transmisión intergeneracional de la pobreza (Díaz, Ludwig y Trivelli, 2009).

De acuerdo con Arroyo (2010), el Programa Juntos es percibido por los beneficiarios como el soporte central para lograr un cambio en su vida personal y familiar. Además, el autor ha identificado algunos desafíos a superar vinculados a los factores de selección de beneficiarios, tales como problemas respecto a los instrumentos para medir la situación de cada familia, problemas de comunicación y traslado de información hacia las comunidades, desplazamiento de familias fuera de sus localidades y pobladores que no se inscribieron inicialmente debido a que dudaban de la veracidad del Programa.

Sobre estos casos existen diversos estudios realizados desde la perspectiva del beneficiario y los impactos que vienen generando los programas sociales, pero hay limitada bibliografía que trata sobre la percepción que tienen los pobladores no beneficiarios de dichos programas, como es Juntos. Solo se 
cuenta con algunos informes sobre evaluaciones de estos programas que reportan cantidades de beneficiarios o usuarios. En ese sentido, el presente estudio constituye un aporte, pues trata acerca de la percepción cultural que tienen los pobladores del Valle del Mantaro sobre el Programa Juntos; recoge las impresiones, de acuerdo al sentir, pensar y actuar, de quienes no son beneficiarios del Programa, teniendo en cuenta las etapas anterior y posterior a su implementación.

Los resultados que se reportan en este artículo servirán de referencia a los principales tomadores de desiciones, como son autoridades y funcionarios que operan en cada uno de estos ámbitos jurisdiccionales.

\section{Método}

El área considerada para el presente estudio fue el Valle del Mantaro, cuatro provincias y ocho distritos: Acolla y Sincos de la provincia de Jauja; Chambará y Orcotuna de la provincia de Concepción; Ahuac y Chongos Bajo de la provincia Chupaca; Sapallanga y Sicaya de la provincia de Huancayo. Los distritos fueron seleccionados teniendo en cuenta la mayor cantidad de beneficiarios de los últimos dos años.

La técnica empleada fue la encuesta, y el instrumento, un cuestionario tipo escala de Likert, el cual fue aplicado a una muestra representativa de 382 personas no beneficiarias del Programa Juntos. Asimismo, se llevaron a cabo entrevistas durante agosto y setiembre del año 2018.

El estudio consideró a varones y mujeres mayores de 15 años de ocho distritos de las cuatro provincias del Valle del Mantaro, puesto que, según la clasificación de Hernández y Christlieb (2013), corresponden a los grupos poblacionales: jóvenes, de 16 a 24 años; adultos, de 25 a 59 años, y adultos mayores, de 60 años a más. Estos pobladores habitan y participan activamente en las diversas actividades familiares y comunales de cada distrito priorizado, los cuales fueron visitados por el equipo de tres investigadores de acuerdo a una programación.

Se realizó una investigación de carácter cuantitativo y cualitativo, de tipo básico y nivel descriptivo; la variable de estudio percepción cultural sobre el Programa Juntos fue descrita, permitiendo así conocer el fenómeno 
estudiado. Como método general, se aplicó el método científico y como método específico, el método descriptivo.

Primero. Para el nivel teórico, se utilizó el método de la investigación bibliográfica, archivística y documental planteada por De la Torre y Navarro (2008); lo cual supuso la revisión de diversos documentos sobre el tema de estudio, libros, revistas y artículos científicos, accesando a diversos repositorios de las principales publicaciones científicas del ámbito nacional e internacional como Google académico, Alicia, Concytec, Scielo, Scopus y otros, para profundizar y ampliar el marco teórico de la investigación.

Segundo. Para obtener información de campo, la técnica utilizada fue la encuesta perfilada según la escala de Likert (1932). El instrumento empleado fue el cuestionario, cuya fiabilidad se determinó a través del alfa de Cronbach, que reportó un coeficiente de 0,86 , teniendo en cuenta que la consistencia interna del instrumento es aceptable a partir de 0,7 y perfecta a 1 . El cuestionario, diseñado para medir opiniones y actitudes, contenía un total de 30 ítems, todos con 5 opciones: muy mala, mala, regular, buena y muy buena. Asimismo, se realizaron entrevistas a algunos dirigentes, funcionarios y autoridades en cada distrito. Estas actividades, en su mayoría, fueron ejecutadas por las estudiantes consideradas como colaboradoras en la presente investigación, bajo el seguimiento y supervisión del docente responsable.

Los datos obtenidos a través de la aplicación del cuestionario fueron procesados con el programa estadístico SPSS, el cual arrojó cifras con un nivel de confianza al $95 \%$ y un margen de error al $6 \%$. Los resultados fueron analizados e interpretados y han permitido al equipo formular conclusiones sobre la percepción del Programa Juntos que tienen los no beneficiarios en los rubros de nutrición, salud y educación.

\section{Resultados}

Percepción cultural de los no beneficiarios sobre el cambio en nutrición, salud y educación de los beneficiarios de Juntos 
Tabla 1

Percepción cultural sobre el cambio en la nutrición de los hijos de los beneficiarios de Juntos

\begin{tabular}{ccccc}
\hline Muy mala & $\begin{array}{c}\text { Antes de } \\
\text { Juntos }\end{array}$ & $\begin{array}{c}\text { Después de } \\
\text { Juntos }\end{array}$ & $\begin{array}{c}\text { Diferencia entre } \\
\text { el antes y el } \\
\text { después }\end{array}$ \\
\hline Málido & 4,2 & 3,9 & $-0,3$ \\
& Regular & 29,1 & 26,2 & $-2,9$ \\
& Buena & 40,1 & 33,8 & $-6,3$ \\
& Muy buena & 26,2 & 34,3 & 8,1 \\
TOTAL & 0,5 & 1,8 & 1,3 \\
\hline
\end{tabular}

En la tabla 1, en el rubro antes de Juntos, se observa que más del $40 \%$ de los pobladores del Valle del Mantaro perciben que la nutrición de los pobladores antes de ser beneficiarios del Programa Juntos era regular; también se aprecia que cerca del 30 \% la califica como mala. Además, existe diferencia significativa entre las cifras de los 5 niveles de percepción.

En el rubro después de Juntos, se observa que casi el $35 \%$ de los pobladores del Valle del Mantaro consideran que hoy en día la nutrición de los pobladores beneficiarios del Programa Juntos es buena, en tanto que cerca del $34 \%$ cree que es regular.

A partir de estos primeros resultados, se puede inferir que la nutrición de las personas beneficiarias de Juntos es mejor de lo que fue antes de la implementación del Programa. Como señala una de las entrevistadas, antes estas personas comían cualquier cosa que se les invitaba y llevaban otra parte para sus hijos(as); hoy en día han mejorado su nutrición porque reciben charlas informativas e instructivas de los diversos programas.

Esto se corrobora con los resultados que reporta el rubro diferencia entre el antes y el después, donde se observa que el nivel de percepción buena ha tenido una mejora significativa de $8.1 \%$, seguido por regular, que presenta un retroceso de $6.3 \%$. 
Tabla 2

Percepción cultural sobre el cambio en el cuidado de la salud de los hijos de los beneficiarios de Juntos

\begin{tabular}{|c|c|c|c|c|}
\hline & & $\begin{array}{c}\text { Antes de } \\
\text { Juntos }\end{array}$ & $\begin{array}{c}\text { Después de } \\
\text { Juntos }\end{array}$ & $\begin{array}{c}\text { Diferencia entre } \\
\text { el antes y el } \\
\text { después }\end{array}$ \\
\hline \multirow{6}{*}{ Válido } & Muy mala & 4,7 & 2,9 & -1.8 \\
\hline & Mala & 32,2 & 13,6 & -18.6 \\
\hline & Regular & 37,4 & 29,6 & -7.8 \\
\hline & Buena & 24,9 & 46,6 & 21.7 \\
\hline & Muy buena & 0,8 & 7,3 & 6.5 \\
\hline & TOTAL & 100,0 & 100,0 & \\
\hline
\end{tabular}

Como se aprecia en la tabla 2, respecto al rubro antes de Juntos, más del $37 \%$ de los pobladores del Valle del Mantaro consideran que la salud de sus compueblanos antes de ser beneficiarios del Programa Juntos era regular; asimismo, más del $32 \%$ cree que era mala. Se observa también que existe diferencia significativa entre las cifras de los 5 niveles de percepción.

De otro lado, en el rubro después de Juntos, se observa que más del $46 \%$ de los pobladores del Valle del Mantaro consideran que hoy en día la salud de los pobladores beneficiarios del Programa Juntos es buena, mientras que casi el $30 \%$ la califica como regular. Se aprecia, además, que existen diferencias significativas entre los 5 niveles de percepción.

Con base en estas cifras, se puede deducir que la salud de las personas beneficiarias de Juntos ha logrado una mejora notable respecto a la época anterior a la llegada del Programa. Así, la mayoría de entrevistados refiere que antes de Juntos, estas personas eran abandonadas hasta por sus propios familiares; tampoco acudían a los puestos o centros de salud a atenderse por falta de dinero. Actualmente, en cambio, se ve que son las primeras en solicitar atención, pues tienen que presentar sus papeletas para seguir siendo beneficiarias del Programa.

Esto se condice con los resultados del rubro diferencia entre el antes y el después, donde se observa que el nivel de percepción buena ha tenido un avance significativo de $21.7 \%$, a la vez que el de mala reporta un retroceso de $18.6 \%$. 
Tabla 3

Percepción cultural sobre el cambio en la educación de los hijos de los beneficiarios de Juntos

\begin{tabular}{ccccc}
\hline Vúla mala & $\begin{array}{c}\text { Antes de } \\
\text { Juntos }\end{array}$ & $\begin{array}{c}\text { Después de } \\
\text { Juntos }\end{array}$ & $\begin{array}{c}\text { Diferencia entre el } \\
\text { antes y el después }\end{array}$ \\
\hline Málido & 3,9 & 2,4 & -1.5 \\
& Megular & 23,0 & 18,3 & -4.7 \\
& Buena & 35,3 & 23,8 & -11.5 \\
& Muy buena & 35,6 & 44,8 & 9.2 \\
& TOTAL & 2,1 & 10,2 & 8,6 \\
\hline
\end{tabular}

Como se muestra en la tabla 3, en el rubro antes de Juntos, más del $35 \%$ de los pobladores del Valle del Mantaro creen que la educación de sus compueblanos antes de ser beneficiarios del Programa Juntos era buena; un porcentaje similar considera que esta era regular.

Así también, en el rubro después de Juntos, se observa que casi el $45 \%$ de los pobladores del Valle del Mantaro consideran que, en la actualidad, la educación de los pobladores beneficiarios del Programa Juntos es buena; mientras que casi el $24 \%$ cree que esta es regular. Además, se aprecia que existe diferencia significativa entre los 5 niveles de percepción.

En función de estos resultados, se puede inferir que la educación de los beneficiarios de Juntos es mejor de lo que era antes de la implementación del Programa. La mayoría de personas entrevistadas así lo creen; aunque los encuestados del distrito de Ahuac, provincia de Chupaca, piensan que la educación de antes era mejor que la de ahora, porque hoy en día los estudiantes de secundaria y algunos de superior no saben redactar oficios ni solicitudes, mientras que los "antiguos" sí sabían hacerlo.

Esto concuerda con los resultados del rubro diferencia entre el antes y el después, donde se observa que el nivel de percepción regular ha tenido una diferencia significativa de $-11.5 \%$ por ciento, seguido por el de buena, que presenta un crecimiento de $9.2 \%$. 


\section{Discusión}

Las percepciones culturales que tienen los pobladores no beneficiarios sobre los beneficiarios del Programa Juntos, que se viene ejecutando en el Valle del Mantaro, están referidas a los cambios que han presentado factores como el cuidado de la salud, la educación y la nutrición de los hijos de los beneficiarios a partir de la implementación del Programa. Así, en cuanto a la percepción de la nutrición, hubo un incremento del $8.1 \%$ en el nivel de buena, con respecto a la situación anterior a la llegada del Programa. La percepción de la salud también experimentó un incremento del 21 \% en el nivel de buena, en relación a la situación previa a Juntos. Similar cambio se evidenció en la percepción de la educación, con un incremento del $9.2 \%$ en el nivel de buena, siempre con respecto al escenario anterior al Programa.

La percepción cultural sobre el cambio en la nutrición de los hijos de los beneficiarios del Programa Juntos tras la llegada del programa presenta mayores porcentajes en los niveles buena (cerca del $35 \%$ ), regular (más del $30 \%$ ) y mala (más del $25 \%$ ); estos, comparados con los niveles de la percepción sobre la situación anterior al Programa, se han reducido en un $6.3 \%, 2.9 \%$ y un $0.3 \%$, respectivamente. A la vez, las percepciones buena y muy buena se han incrementado, pasando de $26 \%$ a $34 \%$ y de $0.5 \%$ a $1.8 \%$, respectivamente. Podemos concluir, entonces, que las percepciones positivas se han incrementado.

Estas percepciones concuerdan con los resultados de diversos estudios (Cavero et al., 2017; Perova y Vakis, 2010; MIDIS, 2017; Segovia y Mira, 2014) que refieren el impacto positivo de Juntos; sin embargo, cabe precisar que el Programa carece de una línea de base, por lo que no se puede medir con precisión el nivel de mejora que genera, solo se especula con datos encontrados o construidos en campo.

Según Sánchez y Jaramillo (2012), la inversión en los primeros años de vida de los seres humanos tiene implicancias a largo plazo, como en el rendimiento de los individuos en el mercado laboral; esto tiene relación con los programas de transferencia condicionada que desarrolla el Estado, caso de Juntos, el cual tiene como uno de sus objetivos la mejora de la nutrición de los niños menores de 5 años.

En cuanto a la percepción cultural sobre el cambio en el cuidado de la 
salud de los hijos de los beneficiarios del Programa Juntos, más del 45 \% de los encuestados considera que la situación de los beneficiarios posterior a la llegada del Programa es buena, mientras que más del $5 \%$ cree que esta es muy buena; de otro lado, cerca del 30 \% piensa que dicha situación es regular. Con respecto a las percepciones culturales sobre el escenario anterior a la implementación del Programa, se observa que los niveles de mala y muy mala se han reducido en $18.6 \%$ y $1.8 \%$, respectivamente; asimismo, la percepción regular ha disminuido en un $7.8 \%$, mientras que las percepciones de buena y muy buena se han incrementado en un $21.7 \%$ y un $6.5 \%$, respectivamente. Por lo tanto, se puede considerar que, en relación al cambio en el cuidado de la salud, la percepción es que este ha mejorado.

Este resultado concuerda con el de Perova y Vakis (2010), quienes, en un estudio realizado para el Banco Mundial, señalan que el Programa Juntos tiene impacto positivo en las áreas de nutrición y salud, ya que se ha evidenciado un incremento significativo de los usuarios del servicio de salud; sin embargo, no abordan las condiciones en las que se hallan los establecimientos, los insumos médicos, los equipos y el mismo personal que brinda los servicios de salud. Debe considerarse que en las zonas rurales, hay insuficiente personal de salud calificado (Mayca, Palacios-Flores, y Medina, 2009).

Por otra parte, los resultados encontrados por Contreras, J. I., Contreras, L. y Rojas (2015) en Chile, sobre la vulneración de derechos hacia niños(as) y adolescentes, indican la ausencia de instrumentos legislativos eficaces o planes de infancia actualizados que permitan articular eficientemente la respuesta del Estado, que carece de una política pública integral para la infancia y familia. Sería importante tomar en cuenta este escenario para el caso peruano.

Similar panorama presenta la percepción cultural sobre el cambio en la educación de los hijos de los beneficiarios del Programa Juntos, pues la percepción dominante es que la situación después de la llegada del Programa es buena, bordeando un $45 \%$. Si bien las percepciones regular y mala son altas, cerca de un $25 \%$ y $20 \%$, respectivamente, y la muy buena solo alcanza un $10 \%$, se debe considerar que, comparadas con la situación anterior a Juntos, los niveles de regular, mala y muy mala se han reducido (en $11.5 \%$, $4.7 \%$ y $1.5 \%$, respectivamente), mientras que los de buena y muy buena se 
han incrementado (en $9.2 \%$ y $8.6 \%$, respectivamente). Por consiguiente, podríamos considerar que las percepciones positivas sobre la educación de los hijos de los beneficiarios se han incrementado.

Los resultados encontrados en el Valle del Mantaro concuerdan con los reportados por otros estudios (Cecchini y Atuesta, 2017; Alcázar, 2009; Díaz et al., 2009; Cavero-Arguedas et al., 2017) que refieren el impacto positivo de Juntos en el sector educación, pues no solo se observa el incremento de los educandos, sino también la reducción del porcentaje de deserción y abandono escolar. No obstante, como señalan los entrevistados, esto ocurre porque los beneficiarios tienen que cumplir el compromiso optado con el Estado para no perder el beneficio.

\section{Conclusiones}

Las percepciones culturales de los no beneficiarios sobre la salud, la educación y nutrición de los beneficiarios del Programa Juntos sugieren la existencia de cambios favorables en dichos factores tras la implementación del Programa. Así, las valoraciones positivas (muy buena y buena) del escenario actual son mayores a las que se tienen de la situación anterior a la llegada del Programa; mientras que las valoraciones negativas (mala y muy mala) son menores. Esto nos permite entender que los pobladores no beneficiarios han visto cambios positivos introducidos en su comunidad por el Programa Juntos; hecho que se corrobora, además, con las entrevistas realizadas, en las cuales se evidencia una mejora en las condiciones de vida de los beneficiarios manifestada en la nutrición, salud y educación.

Este estudio es importante en tanto que permite conocer la valoración que hacen los no beneficiarios del Programa, pues se trata de una visión externa, más objetiva y neutral del problema; diferente a la del beneficiario o a la de los trabajadores del sector, cuyas apreciaciones parecen regirse por un mismo parámetro.

Asimismo, una de las críticas más frecuentes a los programas sociales en su conjunto y no solo a Juntos, es la inexistencia de una línea de base. En este caso, conocer la percepción sobre la situación anterior y posterior a la aplicación del Programa nos permite sostener los cambios acaecidos en la comunidad. 
Finalmente, se han observado ciertas limitaciones para alcanzar los objetivos del Programa; de ahí que sea necesaria una mayor coordinación con las autoridades y otros programas del Estado, pues Juntos no podrá generar mayor impacto si es que no realiza un trabajo coordinado con las autoridades de la localidad, servidores de salud y educación (Morera, 1995) y con las diversas organizaciones presentes en las zonas de intervención.

Agradecimientos: A las autoridades municipales y pobladores de los ocho distritos ubicados en las cuatro provincias del Valle del Mantaro: Acolla, Ahuac, Chambará, Chongos Bajo, Orcotuna, Sapallanga, Sicaya y Sincos, quienes nos brindaron la información requerida para la concreción de la presente investigación.

\section{Referencias bibliográficas}

Alcázar, L. (2009). El gasto público social frente a la infancia. Análisis del Programa Juntos y de la oferta y demanda de servicios asociadas a sus condiciones. Lima: GRADE y Niños del Milenio.

Balbi, F. A. y Boivin, M. (2008). La perspectiva etnográfica en los estudios sobre política, Estado y gobierno. Cuadernos de Antropología Social, 27, 7-17.

Cavero Arguedas, D., De la Vega, V. C., y Cuadra-Carrasco, G. (2017). Effects of social programs on indigent population health: Evidence from resultsbased budgeting's impact evaluations to social programs in Peru. Revista Peruana de Medicina Experimental y Salud Pública, 34 (3), 528537. doi:https://doi.org/10.17843/rpmesp.2017.343.3063

Cecchini, S. y Atuesta, B. (2017). Programas de transferencias condicionadas en América Latina y el Caribe: tendencias de cobertura e inversión. Serie Políticas Sociales 224. Santiago de Chile, CEPAL.

Cecchini, S. y Madariaga, A. (2015). Programas de transferencias condicionadas: balance de la experiencia reciente en América Latina y el Caribe. Santiago de Chile: Naciones Unidas . Recuperado de https:// repositorio.cepal.org/bitstream/handle/1 1362/27854/S201 1032_es.pdf

Contreras, J.I., Contreras, L., y Rojas, V. (2015). Análisis de programasrelacionados con la intervención en niños, niñas y adolescentes vulnerados en sus 
derechos: Ia realidad chilena. Psicoperspectivas. Individuo y Sociedad, 14(1), 89-102. doi: https://doi.org/10.5027/psicoperspectivas-Vol14Issue 1-fulltext-528

Díaz, R., Ludwig, H., y Trivelli, C. (2009). Análisis de la implementación del Programa Juntos en las regiones de Apurímac, Huancavelica y Huánuco. Lima: CIES y CARE Perú.

García, A. (1990). Desarrollo Humano. Informe 1990. Bogotá, Colombia: Tercer Mundo Editores. Recuperado de http://hdr.undp.org/sites/default/files/ hdr_1990_es_completo_nostats.pdf

Guabloche, J. y Sánchez, A. (2011). ¿Qué sabemos sobre los programas de transferencias condicionadas? Moneda, 147, 19-23.

Hernández, J. A. R. y Christlieb, F. F. (2013). Paisaje e identidad en El Arbolito, Pachuca, Hidalgo. Jóvenes y adultos en apego a un barrio ex minero. Investigaciones Geográficas, 80, 71-85.

Lucci, M. J. (2018). Políticas sociales y redes políticas. La implementación de un programa de economía social en un municipio de Argentina. Espiral, Estudios sobre Estado y sociedad, 25(72), 123-147. doi: http://dx.doi. org/10.32870/espiral.v25i72.6526

Mayca, J., Palacios-Flores, E., y Medina, A. (2009). Percepciones del personal de salud y la comunidad sobre la adecuación cultural de los servicios materno-perinatales en zonas rurales andinas y amazónicas de la región Huánuco. Revista Peruana de Medicina Experimental y Salud Pública, 26(2), 145-160.

Ministerio de Desarrollo e Inclusión Social. (2017). Resolución Ministerial N. ${ }^{\circ}$ 278-2017-MIDIS.Manualdeoperaciones delPrograma Juntos.Recuperado de http://www2.juntos.gob.pe/docs/Portal_transparencia/procesos/ Manual_Operaciones/R.M_278-2017-MIDIS_Manual_operaciones.pdf

Ministerio de Desarrollo e Inclusión Social. (2013). Estrategia Nacional de Desarrollo e Inclusión Social "Incluir para crecer". Recuperado de http:// www.midis.gob.pe/dmdocuments/estrategia_incluircrecer.pdf

Morera, N. E. (1995). La Gerencia Social: herramienta indispensable para la conducción de servicios sociales en el umbral del siglo XXI. Revista Costarricense de Trabajo Social, 5. Recuperado de https://revista. trabajosocial.or.cr/index.php/revista/article/view/148/161 


\section{3}

Perova, E. y Vakis, R. (2010). El impacto y potencial delPrograma Juntos en Perú: evidencia de una evaluación no experimental. Lima: Programa Juntos y Banco Mundial. Recuperado de http://repositorio.minedu. gob.pe/bitstream/handle/123456789/3974/El\%20impacto\%20y\%20 potencial\%20del\%20Programa\%20Juntos\%20en\%20Per\%C3\%BA\%20 evidencia\%20de\%20una\%20evaluaci\%C3\%B3n\%20no-experimental. pdf? sequence $=1$ \&isAllowed=y

Rico, M.N. (coord.), Cecchine, S. y Vargas, L. H. (2014). Transferencias de ingresos para la erradicación de la pobreza. Dos décadas de experiencias en los países de la UNASUR. Santiago de Chile: Naciones Unidas.

Rodríguez, C. (2011). Programas de transferencias condicionadas de ingreso e igualdad de género ¿̇Por dónde anda América Latina? Santiago de Chile: Naciones Unidas. Recuperado de https://repositorio.cepal.org/ bitstream/handle/11362/5836/1/S1100854_es.pdf

Sánchez, A. Jaramillo, M. (2012). Impacto del programa Juntos sobre nutrición temprana. Revista Estudios Económicos, 23, 53-66- Recuperado de http://www.bcrp.gob.pe/docs/Publicaciones/Revista-EstudiosEconomicos/23/ree-23-sanchez-jaramillo.pdf

Segovia, P. y Mira, S. (2014). Evaluación de políticas y programas sociales en América Latina: situación actual. Revista de Ciencias Sociales, 20(3), 446-458.

Surrallés, A. (2003). De la percepción en antropología. Algunas reflexiones sobre la noción de persona desde los estudios amazónicos. INDIANA, 20, 59-72.

Vargas, E. (2014). Corresponsabilidad y articulación: una mirada a los pilares de gestión del Programa Juntos (tesis de maestría). Pontificia Universidad Católica del Perú, Lima. Recuperada de http://tesis.pucp.edu.pe/ repositorio/handle/20.500.12404/5801

Vargas, L. M. (1994). Sobre el concepto de percepción. Alteridades, 4(8), 47-53. Recuperado de http://www.redalyc.org/articulo.oa? id=7471 1353004

Vivaldi, F. y Barra, E. (2012). Bienestar psicológico, apoyo social percibido y percepción de salud en adultos mayores. Terapia Psicológica, 30(2), 23 29. doi: https://doi.org/10.4067/S0718-48082012000200002 